\title{
Marmosets prove to be polite conversationalists
}

\author{
Monkeys take it in turns to 'talk' to each other, like humans.
}

\section{Daniel Cossins}

17 October 2013

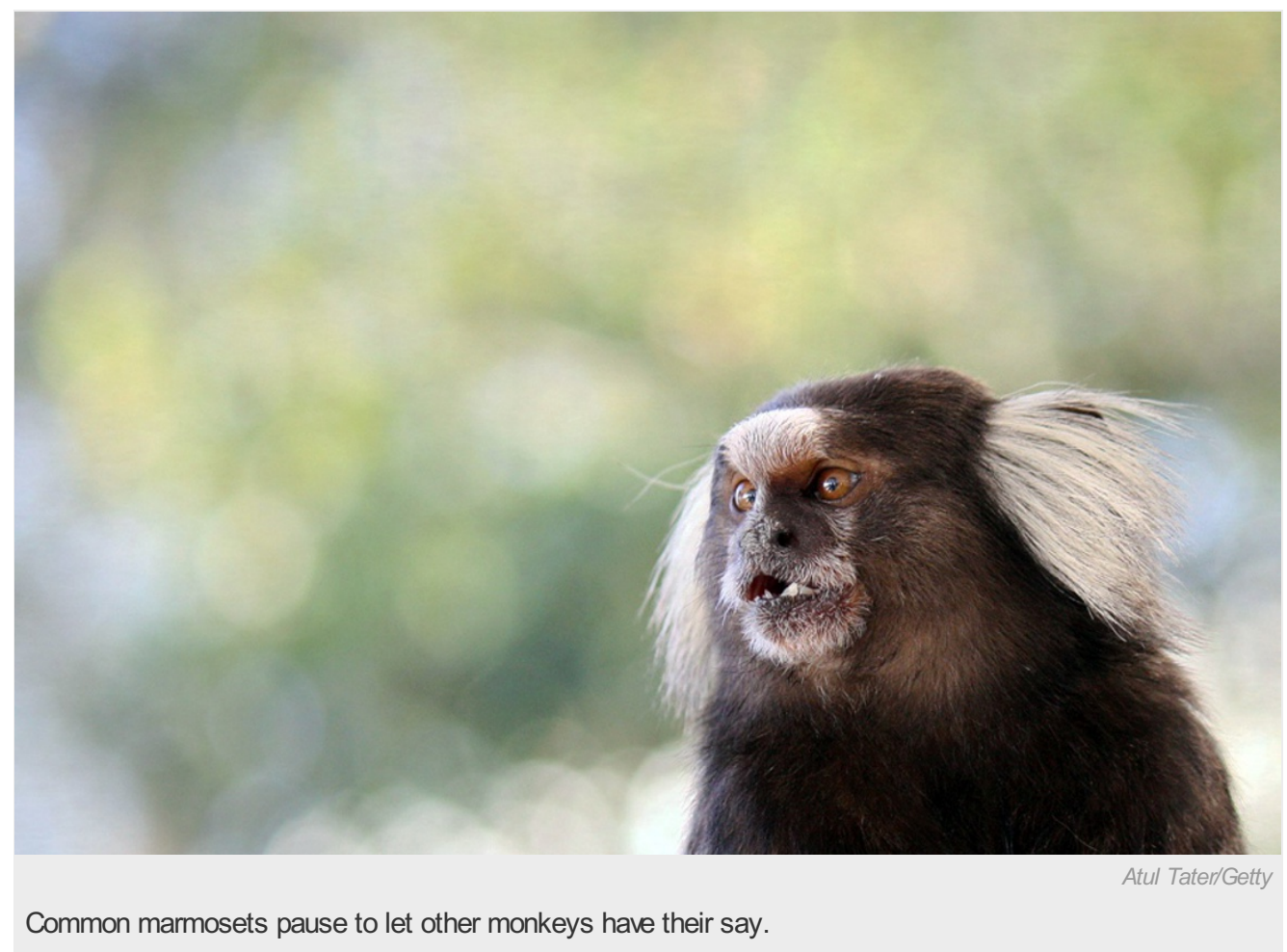

It may not always seem like it, but humans usually take turns speaking. Research published today in Current Biology ${ }^{1}$ shows that marmosets, too, wait for each other to stop calling before they respond during extended vocal exchanges. The discovery could help to explain how humans came to be such polite conversationalists.

Taking turns is a cornerstone of human verbal communication, and is common across all languages. But with no evidence that nonhuman primates 'converse' similarly, it was not clear how such behaviour evolved. The widely accepted explanation, known as the gestural hypothesis, suggests that humans might somehow have taken the neural machinery underlying cooperative manual gestures such as pointing to something to attract another person's attention to it, and applied that to vocalization.

Not convinced, a team led by Daniel Takahashi, a neurobiologist at Princeton University in New Jersey, wanted to see whether another primate species is capable of cooperative calling. The researchers turned to common marmosets (Callithrix jacchus) because, like humans, they are prosocial — that is, generally friendly towards each other — and they communicate using vocalizations.

\section{After you}

The team recorded exchanges between pairs of marmosets that could hear but not see each other, and found that the monkeys never called at the same time. Instead, they always waited for roughly 5 seconds after a caller had finished before responding.

Importantly, the marmosets engaged in these exchanges with any other marmoset. This distinguishes what they are doing from the coordinated calling of birds and frogs, which is confined to pair-bonded duets and male advertisement calls. Furthermore, marmoset calls were entrained to one another - if one monkey increased its calling speed, so did the other. That is exactly what happens in human conversation.

The results demonstrate for the first time that cooperative vocal communication has evolved more than once, offering "a plausible alternative" to the gestural hypothesis, says Asif Ghazanfar, also a neurobiologist at Princeton and a co-author of the paper. Turntaking vocal behaviour may evolve in species that are very social and use calls to communicate. "We can bypass this mysterious jump 
from manual gestures to vocalizations."

Thore Bergman, a biological psychologist at the University of Michigan in Ann Arbor, is intrigued that marmosets coordinate their calls so precisely. "Such coordination is one of the many aspects of human language that are difficult to study evolutionarily because language is so different from what animals do," says Bergman. "Here we have a potential model of the temporal coordination involved in conversations."

Ghazanfar says that his team can now use marmosets as models to investigate the neural mechanisms behind cooperative vocalization, and to study how early-life interactions influence the development of the trait. "One of our ongoing goals is to illuminate communication-deficit disorders in humans," he says.

Nature I doi:10.1038/nature.2013.13974

\section{References}

1. Takahashi, D. Y., Narayanan, D. Z. \& Ghazanfar, A. A. Curr. Biol. http://dx.doi.org/10.1016/j.cub.2013.09.005 (2013). 